\title{
MATHEMATICAL MODEL TO INVESTIGATE THE DRAG REDUCTION OF KEROSENE WITH POLYMER UNDER TURBULENT FLOW \\ Adil Abbas Alwan \\ Adil.Abass@uobabylon.edu.iq
}

\begin{abstract}
This paper present a mathematical study on drag reduction by polymer additive such as poly isobutylene (PIB) with kerosene in turbulent pipe flow by using computational fluid dynamic commercial package program (COMSOL 4.4) solution. Theoretically the computational study was used to calculate the pressure drop in two dimensions geometric model with $6 \mathrm{~m}$ length and $80 \mathrm{~mm}$ width as a diameter of the pipe, Geometric shape has been drawing by tools of the program windows, and to simulated the flow region mathematically the flow region is divide into very small parts (mesh generation). The model that used in the mathematical modelling method was $(\mathrm{k}-\varepsilon)$ mathematical turbulent model to study the internal pipe flow properties. The continuity and momentum equations and two $\mathrm{k}-\varepsilon$ model equations have been solved by the program to obtain the theoretical results. There variables that used in the theoretical study were effective density, effective viscosity, inlet velocity, and outlet pressure. The boundary condition was inlet and outlet velocity, temperature, and pressure of flow, and the velocity $(\mathrm{u}=0)$ at the pipe wall. The theoretical calculations show that the velocity and drag reduction percentage increases with polymer concentration and volume flow rate increasing where maximum DR\% is $15.8 \%$ at volume flow rate $500 \mathrm{l} / \mathrm{min}$ with polymer concentration $100 \mathrm{ppm}$, pressure drop decreases with polymer concentration increasing. Friction factor decreases with polymer concentration increased, also shear stress decrease with polymer concentration increasing.
\end{abstract}

KEY WORDS: Kerosene flows, polymer additive, pressure drop, drag reduction.

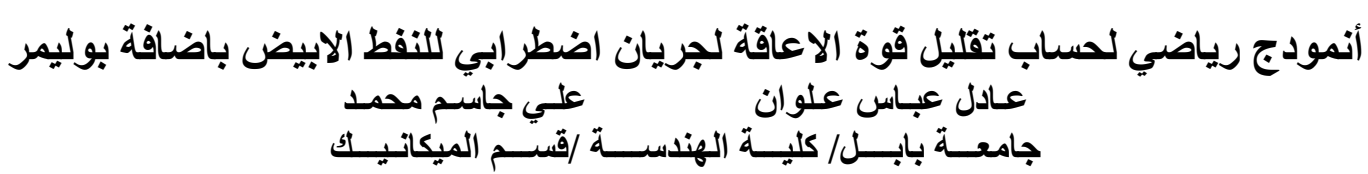

الخلاصة

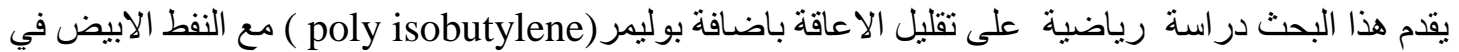

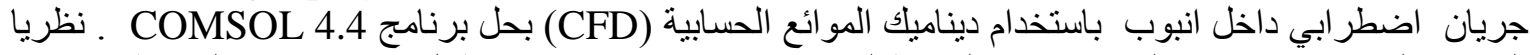

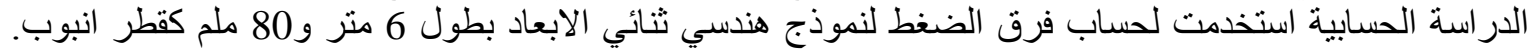

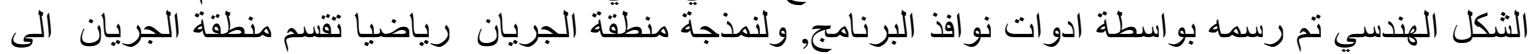

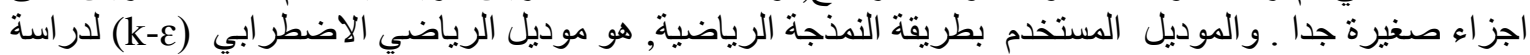

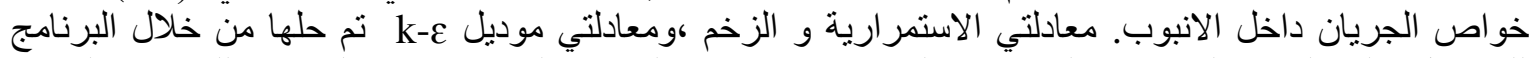

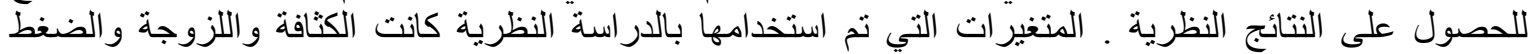

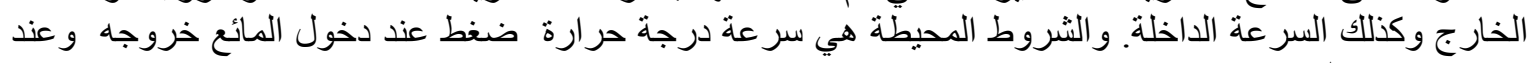

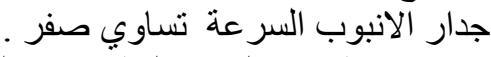

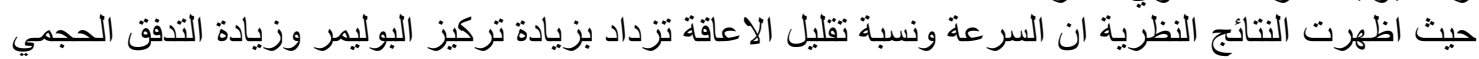

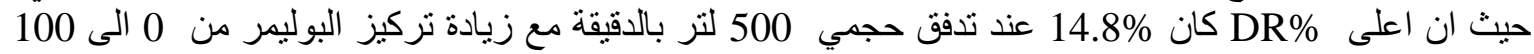

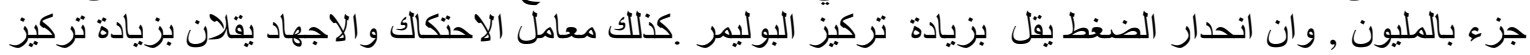


NOMENCLATURE

\begin{tabular}{|c|c|c|}
\hline Symbol & Meaning & Units \\
\hline$D R$ & Drag Reduction & --- \\
\hline$g_{x}, g_{y}, g_{z}$ & Acceleration Components on Cartesian Coordinates & $\mathrm{m} / \mathrm{s}^{2}$ \\
\hline$k$ & Turbulent Kinetic Energy & $\mathrm{N}^{2} / \mathrm{s}^{2}$ \\
\hline$k_{c s}$ & Thermal Conductivity of Carbon Steel Pipe & $\mathrm{W} / \mathrm{m} .{ }^{\circ} \mathrm{C}$ \\
\hline$k_{p}$ & Thermal Conductivity of Pyrex Plastic Pipe & $\mathrm{W} / \mathrm{m} .{ }^{\circ} \mathrm{C}$ \\
\hline$\Delta p_{n}$ & Pressure drop of pure kerosene & $\mathrm{N} / \mathrm{m}^{2}$ \\
\hline$\Delta p_{a}$ & Pressure drop with polymer additive & $\mathrm{N} / \mathrm{m}^{2}$ \\
\hline$Q$ & Volume Flow Rate & $\ell / \mathrm{min}$ \\
\hline$u$ & Velocity of Flowing Fluid & $\mathrm{m} / \mathrm{s}$ \\
\hline$v_{r} v_{\theta} v_{z}$ & Velocity Components on Cartesian Coordinates & $\mathrm{m} / \mathrm{s}$ \\
\hline$r, \theta, z$ & Cartesian Coordinates & $\mathrm{M}$ \\
\hline$\varphi$ & degree of freedom. & --- \\
\hline
\end{tabular}

\begin{tabular}{|c|c|c|}
\hline \multicolumn{3}{|c|}{ Greek Symbols } \\
\hline$\tau$ & Shear Stress & $\mathrm{N} / \mathrm{m}^{2}$ \\
\hline $\mathcal{E}$ & Dissipation Rate of Turbulent Kinetic Energy & $\mathrm{m}^{2} / \mathrm{s}^{3}$ \\
\hline$\rho$ & Density of Working Fluid & $\mathrm{kg} / \mathrm{m}^{3}$ \\
\hline$\rho_{e}$ & Effective Density of Working Fluid & $\mathrm{kg} / \mathrm{m}^{3}$ \\
\hline$\mu$ & Dynamic Viscosity & $\mathrm{kg} / \mathrm{m} . \mathrm{s}$ \\
\hline$\mu_{e}$ & Effective Dynamic Viscosity & $\mathrm{kg} / \mathrm{m} . \mathrm{s}$ \\
\hline$\mu_{e f}$ & Dynamic Viscosity of Working Fluid & $\mathrm{kg} / \mathrm{m} . \mathrm{s}$ \\
\hline$\mu_{t}$ & Turbulent Dynamic Viscosity & $\mathrm{kg} / \mathrm{m} . \mathrm{s}$ \\
\hline
\end{tabular}

\section{INTRODUCTION}

The drag reduction phenomenon in turbulent fluid flow through a circular pipe due to certain polymer additives with high molecular weight and linear chain, this drag reduction phenomenon is very useful in hydrodynamic mechanical application where reducing the frictional pressure drop and shear stress of the fluid flow through a pipe, also fluid flow increasing. The technologies due to using full scale models in the experimental side led to increase the cost and consumption of time during operating on drag reduction studies, because of it need to measurement equipment's with high expensive and difficult operational conditions. So that; the work Computational Fluid Dynamics (CFD) used to simulate the hydrodynamics of liquid flow in horizontal pipe to overcome these expensively and difficulties in experimental field, then the theoretical work in this study was achieved mathematically to simulate the flow region in (CFD) by using COMSOL 4.4 package program was noted by (Neamah , 2012)

(Cruz et al., 2004) developed a model for turbulent wall flows of viscoelastic fluid at low Reynolds number for $(\mathrm{k}-\varepsilon)$ turbulence model. The model used a non-linear molecular viscosity that is affected by the turbulent fluctuation and a new damping function was introduced to account for near-wall effects. This new function was made equal to the eddy viscosity damping function which was derived 
taking into account viscometeric and elastic effects. Compression of turbulent kinetic energy is not enough good, but the model is able to capture the shift of the peak turbulence kinetic energy and rate of dissipation away from the wall. The decrease in those peak values and of the production of $(\mathrm{k})$, and Reynolds shear stress deficit across the pipe. Further developments of the turbulence model are suggested at the end. Min et.al., 2003) studied the turbulent drag reduction by polymer additives in a channel using direct numerical simulation. The dilute polymer solution is expressed with an Oldroyd-B model that shows a linear elastic behavior. Simulations are carried out by changing the Weissenberg number at Reynolds number of 4000 and 20000 based on the bulk velocity and channel height .The onset criterion for drag reduction predicted in the present study shows a good agreement with previous theoretical and experimental studies. In addition, the flow statistics such as the r.m.s. velocity fluctuations are also in good agreement with previous experimental observation. The onset mechanism of drag reduction is interpreted based on elastic theory, which is one of the most plausible hypotheses suggested in the past. The transport equations of kinetic and elastic energy are derived for the first time. It is observed that the polymer stores the elastic energy from the flow very near the wall and then releases it there when the relaxation time short, showing on drag reduction. However, when the relaxation time is long enough, the elastic energy stored in the very near- wall region is transported to and released in the buffer and log layers, showing a significant amount of drag reduction.

(Paschkewitz et.al., 2004) presented a study of the drag reduction induced by rigid fibres in a turbulent channel flow by using direct numerical simulation. The extra stresses due to the fibers are calculated with the well-known constitutive equation involving the moments of the orientation vector. Drag reductions of up to $26 \%$ are calculated, with the largest drag reductions observed using non-Brownian fibres and semi-dilute concentration. These findings suggest that the elasticity is not necessary to achieve turbulent drag reduction. Flow statistics show trends similar to those observed simulation of polymeric drag reduction: Reynolds stress are reduced, velocity fluctuations in the well-normal and spanwise direction are reduced while streamwise fluctuations are increased, and streamwise vorticity is reduced. They observed strong correlations between the fibre stresses and inter-vortex extensional flow regions. Based on these correlations and instantaneous visualizations of the flow field, they propose a mechanism for turbulent drag reduction by rigid fibre additives

(Dubief et.al. ,2004) showed the numerical simulations of turbulent polymer solutions by using FENE-P model are used to characterize the action of polymers on turbulence in drag reduction flows. The energetics of turbulence was investigated by correlating the work done by polymer on the flow with turbulent structures. Polymers are found to store and to release energy to the flow in well-organized manner. The storage of energy occurs around near-wall vortices as has been anticipated for long time. Quite unexpectedly, coherent release of energy is observed in the very nearwall region. Large fluctuations of polymer work are shown to re-energize decaying streamwise velocity fluctuations in high speed streaks just above the viscous sub layer.

In the present work the theoretical analysis that will be used can simulate the turbulent flow model inside a circular pipe with different concentration of additive and effect on the drag reduction. COMSOL 4.4 was used to solve $\mathrm{k}-\varepsilon$ mathematical turbulent model. The momentum equation is resolved in the domain, continuity equation was solved, and in the momentum equation a gravitational force and surface tension of body forces are taken. The momentum equation depended on the volume of the liquid by the properties $(\mu, \rho)$. The Reynolds averaging for turbulence modeling which is applied the NavierStokes equations 


\section{MATHEMATICAL MODEL}

There are many steps in the implementation of the theoretical study before getting the result from the CFD (COMSOL 4.4) solution. The geometry was a (2D) dimensions model with actual length $6 \mathrm{~m}$ and width $0.08 \mathrm{~m}$ represent a diameter of the pipe. There are available tools at the program window was used to draw demanded geometric shape. Then, the mathematical modelling method of the CFD to simulated the flow region was achieved by divided it into small cells and the condition of the flow can kept constant or slightly varies. The model that has been used for the flow inside a pipe was $\mathrm{k}-\varepsilon$ model because it suits for internal pipe flow. The continuity, momentum differential, and $\mathrm{k}-\varepsilon$ model equation represented by variables at any position on the pipe. Iteratively these equations solved until the solution reached to high accuracy. Therefor the mesh step is very important; there are two types of mesh in this software (userdefined and physics-controlled), the better of this mesh is physics-controlled to reach the convergent solution, where it has needed very high processer computer. The numbers of domain mesh element effect on the result of the drag reduction percentage. There are many cases for the mesh generation during divided the flow region into small cells, the mesh generation for many cases which different in number of domain mesh elements and number of boundary mesh elements as illustrated in Fig.1. The range of mesh from low number to the large number of element about (40000-250000), such as the coarser mesh, coarse mesh, normal mesh, and the fine mesh this effected on the drag reduction that calculated theoretically by the COMSOL program, where the drag reduction increases with increasing of the number of domain mesh elements as shown in Fig.1.

\section{Assumption of Fluid Flow}

There are some assumptions of the fluid flow inside the pipes with additive by application of the COMSOL program analysis such as follows:

1- Incompressible fluid flow.

2- Steady state fluid flow.

3- The fluid flow is turbulent.

4- There is no additional force.

5 - The solution of (kerosene- polymer) is homogenous through all the regions of the pipe.

6- The viscosity of the solution is constant with time.

\section{Boundary Condition}

The boundary conditions can be described in this research as shown in Fig.2. The velocity of kerosene at pipe inlet $(\mathrm{u})$ is considered according to the volume flow rate which are $0.87,1.55,1.77$, and $1.9 \mathrm{~m} / \mathrm{s}$. while at the pipe wall the components of the velocities in $\mathrm{x}, \mathrm{y}, \mathrm{z}$ - directions are $(\mathrm{u}=0$ $\mathrm{v}=0, \mathrm{w}=0$ ). The right of domain the outlet pressure in this experimental was $\mathrm{P}_{\text {out }}=18.5,19.5,20,21$, and $\mathrm{kPa}$. From the windows of the COMSOL4.4, it was choice the turbulent flow and selecting k- $\varepsilon$ turbulent model with selecting determining inlet direction and the velocity of the kerosene from 0.87 to $1.0 \mathrm{~m} / \mathrm{s}$ depending on the volume flow rate values, then choosing outlet by clicking another one and determining outlet direction with outlet pressure $\left(\mathrm{P}=\mathrm{P}_{\text {out }}\right)$ from 18.5 to $21 \mathrm{kPa}$, this values feeding to the COMSOL program to getting results.

\section{Governing Equations}

The solved governing equations in the theoretical work was the continuity, momentum equation in three dimensions that which used in the COMSOL 4.4 program for the theoretical work to obtain on the theoretical results is continuity, and momentum equation, these are in three dimensions. The pressure 
drop was predicted by momentum equation and the velocity estimated by continuity equation. to solving the problem some terms must be eliminated and the time dependent terms must be eliminated for the steady state analysis. Hence, the final form in three dimensional steady state continuity and momentum described by (Yuan, 1970) as:

The continuity equation:

$\frac{\partial r V_{r}}{r \partial r}+\frac{\partial V_{\Theta}}{r \partial \theta}-\frac{\partial V_{z}}{\partial z}=0$

The momentum equations: equation (2) in r- direction, (3) in $\Theta$ - direction, and (4) in z- direction

$\rho_{e}\left(\mathrm{v}_{r} \frac{\partial V_{r}}{\partial r}+\frac{\mathrm{V}_{\Theta}}{r} \frac{\partial V_{r}}{\partial \theta}+\mathrm{v}_{z} \frac{\partial V_{r}}{\partial z}\right)-\frac{V_{\Theta}}{r}=-\frac{\partial P}{\partial r}+\frac{\partial}{\partial r}\left(\mu_{e} \frac{\partial V_{r}}{\partial r}\right)+\frac{1}{r^{2}} \frac{\partial}{\partial \theta}\left(\mu_{e} \frac{\partial V_{r}}{\partial \theta}\right)+\frac{\partial}{\partial z}\left(\mu_{e} \frac{\partial V_{r}}{\partial z}\right)$

$-\frac{2}{r^{2}}\left(\mu_{e} \frac{\partial V_{\Theta}}{\partial \Theta}\right)+\mu_{e} \frac{\partial V_{r} \mu_{e}}{r \partial r}-\mu_{e} \frac{V r}{r^{2}}$

$\rho_{e}\left(v_{r} \frac{\partial V_{\Theta}}{\partial r}+\frac{V_{\Theta}}{r} \frac{\partial V_{\Theta}}{\partial \theta}+v_{z} \frac{\partial V_{\Theta}}{\partial z}\right)+\frac{V r V \Theta}{r}=-\frac{\partial P}{r \partial \theta}+\frac{\partial}{\partial r}\left(\mu_{e} \frac{\partial V_{\Theta}}{\partial r}\right)+\frac{\partial}{r^{2} \partial \theta}\left(\mu_{e} \frac{\partial V_{\Theta}}{\partial \theta}\right)+\frac{\partial}{\partial z}\left(\mu_{e} \frac{\partial V_{\Theta}}{\partial z}\right)+$

$\frac{2}{r^{2}} \frac{\partial V_{r}}{\partial \Theta}+\frac{1}{r} \frac{\partial V_{\Theta} \mu_{e}}{\partial r}-\mu_{e} \frac{V_{\Theta}}{r^{2}}$

$\rho_{e}\left(v_{r} \frac{\partial V_{Z}}{\partial r}+\frac{V \Theta}{r} \frac{\partial V_{z}}{\partial z}+v_{z} \frac{\partial V_{Z}}{\partial z}\right)=-\frac{\partial P}{\partial z}+\frac{\partial}{\partial r}\left(\mu_{e} \frac{\partial V_{z}}{\partial r}\right)+\frac{\partial}{r^{2} \partial \theta}\left(\mu_{e} \frac{\partial V_{z}}{\partial \theta}\right)+\frac{\partial}{\partial z}\left(\mu_{e} \frac{\partial V_{z}}{\partial z}\right)+$

$\frac{\partial V_{z} \mu_{e}}{r \partial z}$

For the turbulent case the effective viscosity

$$
\mu_{e f}=\mu_{e}+\mu_{t}
$$

In present numerical simulation, the $\mathrm{k}-\varepsilon$ turbulent model will be used, which is suitable for internal pipe flow and at the cost of less computational time. In this turbulence model, the eddy $\left(\mu_{t}\right)$ presented in the terms of turbulent kinetic energy dissipation rate $(\varepsilon)$ as follow:

$$
\mu_{t}=\rho_{e} C_{\mu} \frac{k^{2}}{\varepsilon}
$$

COMSOL 4.4 was used to solve the partial differential equations in the two equations model with turbulent kinetic energy $\underline{\mathrm{k}}$ and turbulent kinetic energy dissipation rate $\underline{\varepsilon}$ are detailed by COMSOL user (manual 4.4) as:

$\frac{\partial\left(\rho_{e} V_{r} k\right)}{\partial r}+\frac{\partial\left(\rho_{e} V_{\Theta} k\right)}{r \partial \theta}+\frac{\partial\left(\rho_{e} v_{z} k\right)}{\partial z}=\frac{\partial}{\partial r}\left(\frac{\mu_{t}}{\sigma k} \cdot \frac{\partial k}{\partial r}\right)+\frac{\partial}{r^{2} \partial \theta}\left(\frac{\mu_{t}}{\sigma k} \cdot \frac{\partial k}{\partial \theta}\right)+\frac{\partial}{r \partial z}\left(\frac{\mu_{t}}{\sigma k} \cdot \frac{\partial k}{\partial z}\right)+$

$\mu_{t} \varphi-\rho_{e} \varepsilon$ 


$$
\begin{gathered}
\frac{\partial\left(\rho_{e} V_{r} \varepsilon\right)}{\partial r}+\frac{\partial\left(\rho_{e} V_{\theta} \varepsilon\right)}{r \partial \theta}+\frac{\partial\left(\rho_{e} V_{z} \varepsilon\right)}{\partial z}=\frac{\partial}{\partial r}\left(\frac{\mu_{t}}{\sigma \varepsilon} \cdot \frac{\partial \varepsilon}{\partial r}\right)+\frac{\partial}{r^{2} \partial \theta}\left(\frac{\mu_{t}}{\sigma \varepsilon} \cdot \frac{\partial \varepsilon}{\partial \theta}\right)+\frac{\partial}{r \partial z}\left(\frac{\mu_{t}}{\sigma \varepsilon} \cdot \frac{\partial \varepsilon}{\partial z}\right)+ \\
\mathrm{C}_{1} \mu_{t} \frac{\varepsilon}{k} \varphi-\rho_{e} \mathrm{C}_{2} \frac{\varepsilon^{2}}{k}
\end{gathered}
$$

Virtual values of the various constants in this model are shown in table.1

\section{COMSOL Results Validation}

This program used to solve the partial differential equations with high technique in the field of pressure drop, velocity, and DR\% in turbulent fluid flow, and is focused on flow additives. The number of degrees of freedom of the flow 3 , and can be captured by DNS is limited by the available computational power, and the number of mesh elements, then the work on this program achieve by personal computer with an incepted processor and memory. The result of COMSOL program of drag reduction with concentration for (PIB) was validated by the results of theoretical work of (Shahath, 2015). As shown in Fig.3, where the results give accepted agreement between the theoretical results of this work and theoretical results of (Shahath).

\section{RESULTS AND DISCUSSION}

This mathematical investigation gives the relationship between the concentration of polymer additive with drag reduction pipe flow and the effect of polymer concentration with different temperature on the pressure drop, shear stress, and friction factor. The theoretical results that obtain from the program that by feeding the inlet velocity and outlet pressure which was measured by ultrasonic flow meter and pressure transducer device respectively in experimental work of the same subject, with changing the polymer concentration from 0 to $100 \mathrm{ppm}$, then the inlet velocity that feeding to the program from 0.087 to $1.9 \mathrm{~m} / \mathrm{s}$, and the outlet pressure feeding to the program from 18.5 to $21 \mathrm{kPa}$ which represent in the boundary condition as shown in Fig.2 to getting the theoretical results from the COMSOL program. Pressure and velocity distribution predicted from the results of the program by using the (momentum and continuity) equations. These results were used to calculate the shear stress, friction factor, and the drag reduction percentage by the equations as:

$\Delta p_{i}=P_{i+1}-P_{i}$

Where $\mathrm{i}=1,2,3 \ldots$

$$
\begin{aligned}
& D R \%=\left(\frac{\Delta P_{N}-\Delta P_{a}}{\Delta P_{N}}\right) \\
& f=\frac{\tau}{\frac{1}{2} \rho u^{2} \text { wo.flu. }} \\
& \tau=\left(\frac{\Delta P}{\Delta z}\right)\left(\frac{D}{4}\right)
\end{aligned}
$$

Fig.4 obtains from the difference between the total mean pressure value at distance $0.5 \mathrm{~m}$ and the total mean pressure at distance 5.5 on the pipe for all values of volume flow rate. It has been the pressure drop was increased with increasing the velocity. High increasing was noted after the value of velocity 1.7 $\mathrm{m} / \mathrm{s}$. Also, the pressure drop proportional inversely with working fluid concentration. 
Fig.5 shows the effect of velocity on wall shear stress for different polymer concentrations in pipe flow. It can be noted that the wall shear stress increases with increasing the flow velocity and increasing with the polymer concentration. The shear stress decreases until reach minimum value $0.034 \mathrm{kPa}$ at velocity 0.87 and concentration equal $0 \mathrm{ppm}$. For $80 \mathrm{~mm}$ inside diameter the wall shear stress increases with the velocity until reach maximum value $0.0516 \mathrm{kPa}$ at velocity $1.9 \mathrm{~m} / \mathrm{s}$ for flow of pure kerosene at temperature $47^{\circ} \mathrm{C}$ and time of 90 minutes. For polymer added to the flow with concentration $100 \mathrm{ppm}$ the wall shear stress drops until value $0.0468 \mathrm{kPa}$ at the same velocity $1.9 \mathrm{~m} / \mathrm{s}$. This result was derived from the mean pressure gradient which was presented the pressure drop between the distance $0.5 \mathrm{~m}$ and $5.5 \mathrm{~m}$. This result also was depended on the pipe diameter. These results approximately agreement with the theoretical results of (Chung Yin, 2011). FigURE(6) represents the relation between the friction factor and velocity. These values were depending upon the values of shear stress through the flow velocity for different polymer concentrations and time of 90 minutes with average temperature $47^{\circ} \mathrm{C}$. It is shown that the friction factor decreases with increasing the velocity, and decreases with increasing additive of polymer concentration. It was found at inlet velocity $0.87 \mathrm{~m} / \mathrm{s}$ and distance $0.5 \mathrm{~m}$ a high value of friction factor which was about 0.000116 for pure kerosene for polymer concentration $100 \mathrm{ppm}$ the value is 0.000101 . These reduction values were happened through the turbulent flow of the fluid through the pipe. It was imposed from 0.0000341 for pure kerosene to 0.0000293 at concentration of polymer $100 \mathrm{ppm}$. This was happened at value of velocity $1.9 \mathrm{~m} / \mathrm{s}$. These results approximately agreement with the theoretical results of (ChungYin , 2011). Figures 7 to 9 give the relation between the drag reduction with the velocity of the fluid flow for three polymer concentrations 50,75, and $100 \mathrm{ppm}$ with different values of temperature in different intervals time of flow. These figures show that the drag reduction percentage increases with increasing the temperature values through different period of flow time. The temperature was increased through the flow time. This is due to high friction factor of kerosene and the chemical properties of its components. Maximum drag reduction percentage values were calculated at polymer concentration $100 \mathrm{ppm}$ as shown in Fig. 9. These results were reported as follow: At velocity value $0.87 \mathrm{~m} / \mathrm{s}$ the drag reduction was $12.2 \%$ at temperature $35^{\circ} \mathrm{C}$, to $14 \%$ at temperature $50{ }^{\circ} \mathrm{C}$. It was about $14.4 \%$ at temperature $35^{\circ} \mathrm{C}$ to $16.5 \%$ at temperature $50^{\circ} \mathrm{C}$ with velocity $1.55 \mathrm{~m} / \mathrm{s}$. when the value of velocity $1.77 \mathrm{~m} / \mathrm{s}$, it was $15.7 \%$ at $35^{\circ} \mathrm{C}$ to $17.9 \%$ at temperature $50^{\circ} \mathrm{C}$. Finally, at value of velocity 1.9 $\mathrm{m} / \mathrm{s}$, it was $16.2 \%$ at temperature $35^{\circ} \mathrm{C}$ to $18.4 \%$ at temperature $50^{\circ} \mathrm{C}$. These results are confirmed with the theoretical results of (Hadri and Guillou , 2010). Figure(10) demonstrates that a gradual increasing drag reduction percentage with increasing the polymer concentrations for different values of volume flow rate at time 90 minutes. It was pointed that the drag reduction increases from $4.2 \%$ to $10.9 \%$ for polymer concentration increasing from 50 to $100 \mathrm{ppm}$ at volume flow rate $200 \mathrm{l}$ min. This figure was exposed that the percentage of drag reduction increases with increasing of polymer concentration and volume flow rate. The drag reduction increases from $5.4 \%$ to $12.8 \%$ at volume flow rate $300 \mathrm{l} / \mathrm{min}$, while the increasing values in drag reduction were $5.9 \%$ to $13.9 \%$, and from $6.4 \%$ to $15.8 \%$ at volume flow rate 400, and $500 \mathrm{l}$ /min respectively. This result gave a good agreement with theoretical result of (Sher and Hetsroni, 2008).

\section{CONCLUSIONS}

1. Theoretical analysis was simulated the turbulent flow model inside a circular pipe with different concentration of polymer additive and shows the effect on the drag reduction. Using COMSOL 4.4 package program and using $(\mathrm{k}-\varepsilon)$ turbulent model to solve this analysis. The inlet velocity and the outlet pressure are feeding as known parameters in the continuity and momentum equations to get the results. 
2. The pressure drop and pressure gradient decreases with increasing value of polymer concentration, and increases with increasing the flow of kerosene mean velocity.

4. The mean velocity of flow increases with distance along the pipe, and also increases with increasing the polymer concentration, where the inlet velocity feeding to the program as known parameter.

5. The shear stress and friction factor of kerosene flow estimate from the pressure drop that which producing from the program results.

6. The drag reduction percentage increases with increasing polymer concentration, and mean velocity increasing.

7. It was found the theoretical results were given more reliable results and were coincided with experimental results of (Mohammed, A.J, 2017).

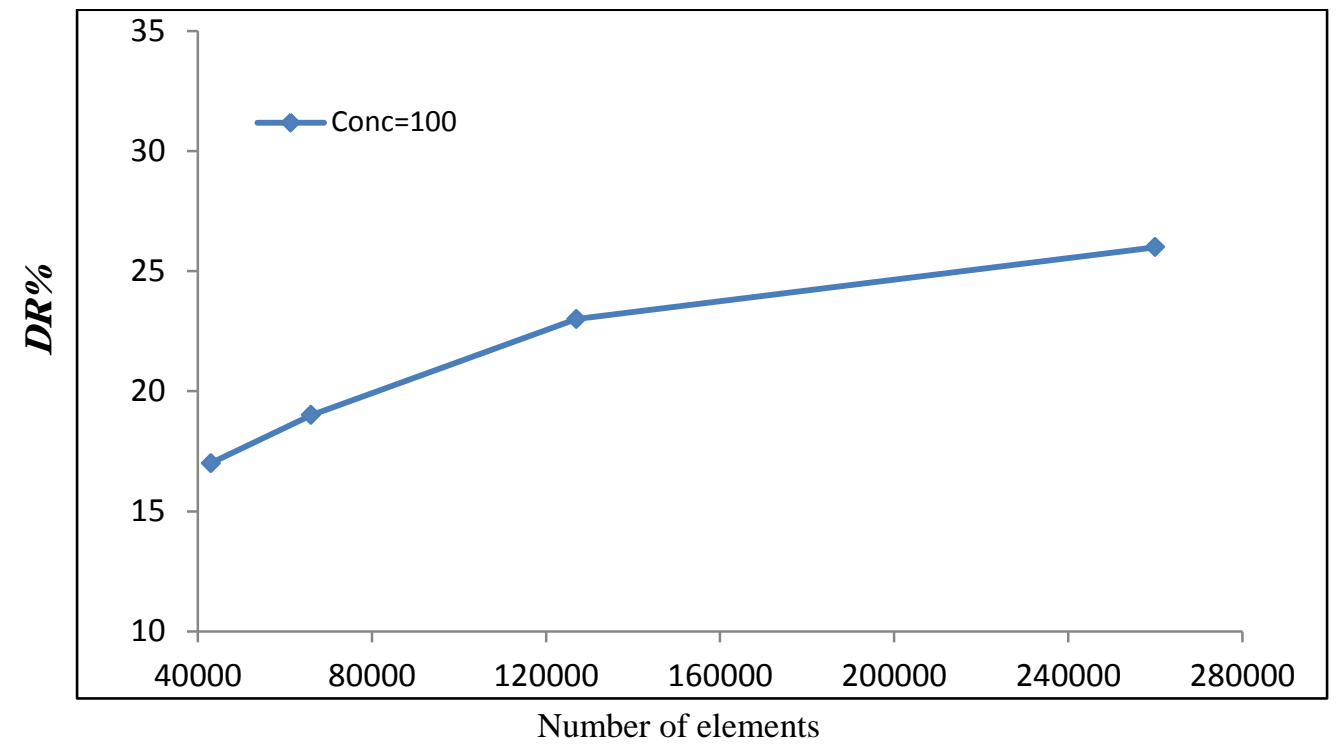

Fig.1 effect the number of mesh element on drag reduction

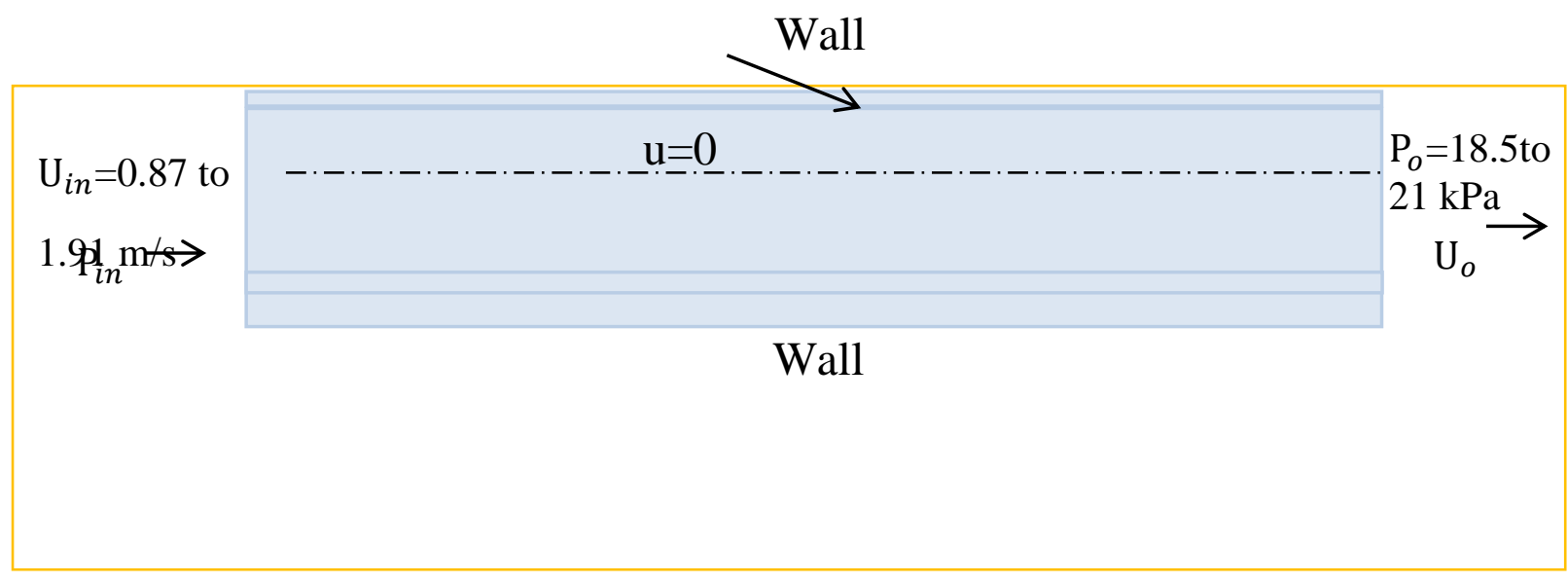

Fig.2 the boundary condition of the pipe

Table 1 Constants for $\mathrm{k}-\varepsilon$ turbulent model

\begin{tabular}{|c|c|c|c|c|}
\hline$\sigma_{k}$ & $\sigma_{\varepsilon}$ & $\mathrm{C}_{1}$ & $\mathrm{C}_{2}$ & $\mathrm{C}_{\mu}$ \\
\hline 1 & 1.3 & 1.44 & 1.92 & 0.09 \\
\hline
\end{tabular}




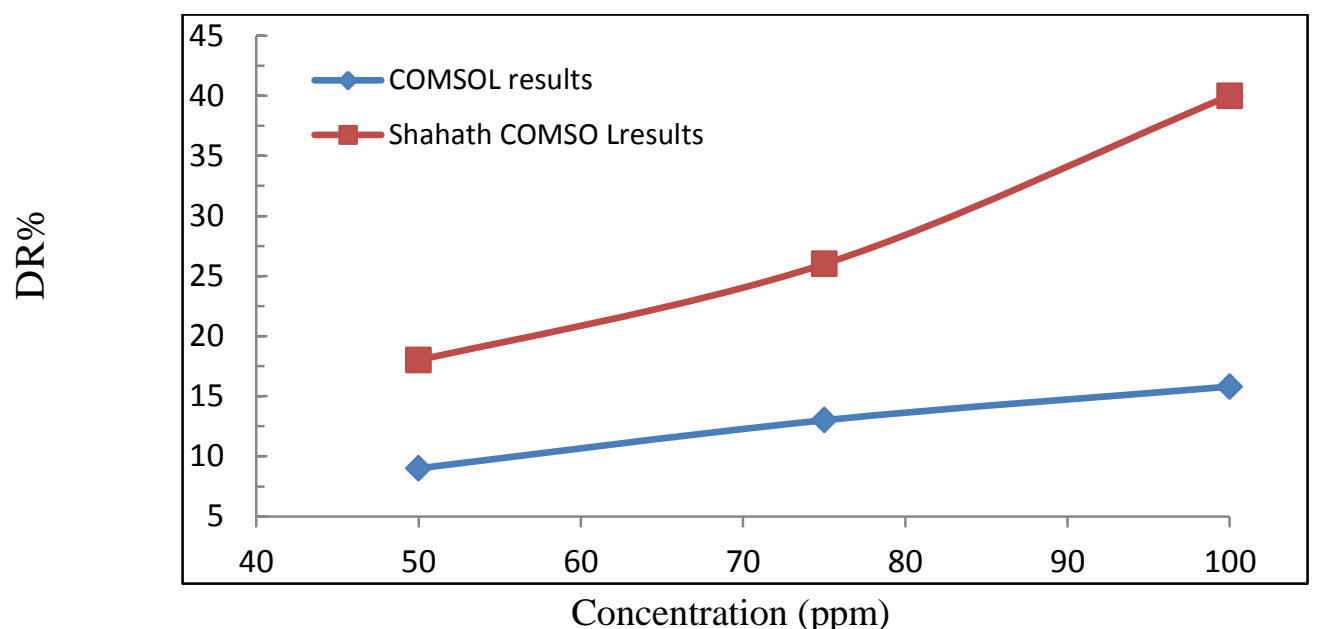

Fig.3 comparison of theoretical COMSOL results with results of Shahath for different polymer concentration

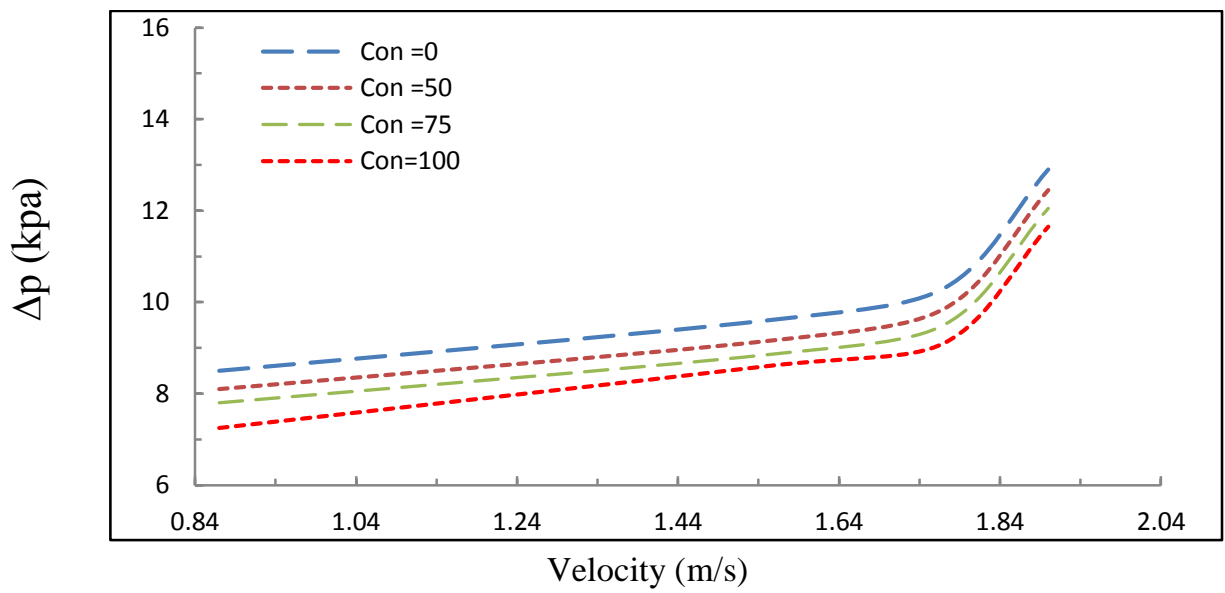

Fig.4 pressure drop with mean velocity for different polymer concentration, at time $=90$ minutes

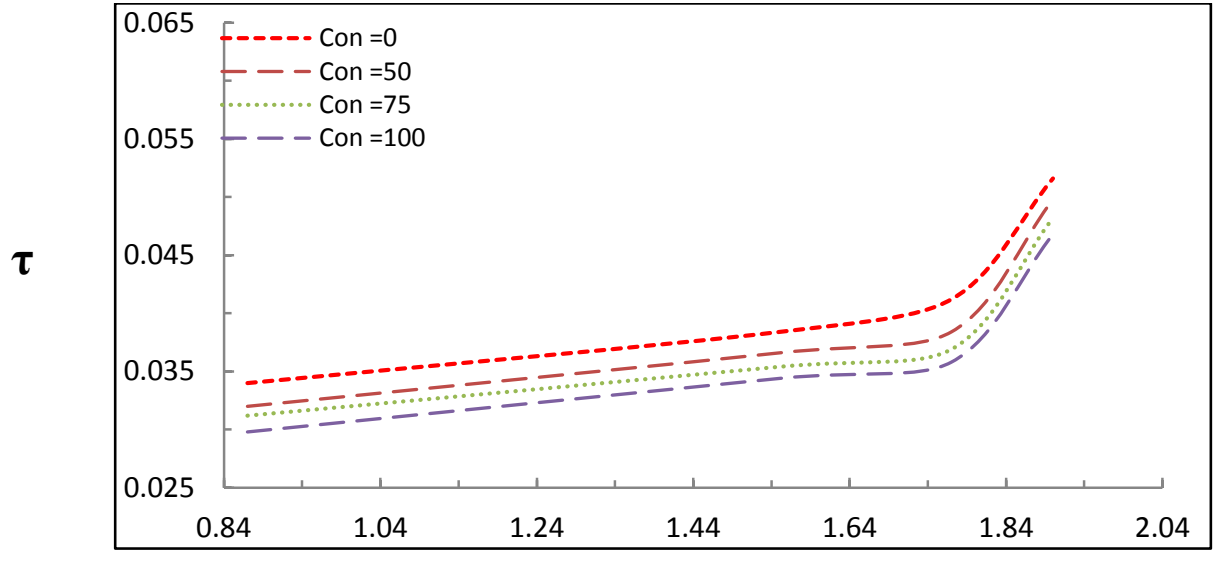

Velocity $(\mathrm{m} / \mathrm{s})$

Fig.5 shear stress with mean velocity for different polymer concentration $(\mathrm{ppm})$, at time $=90$ minutes 


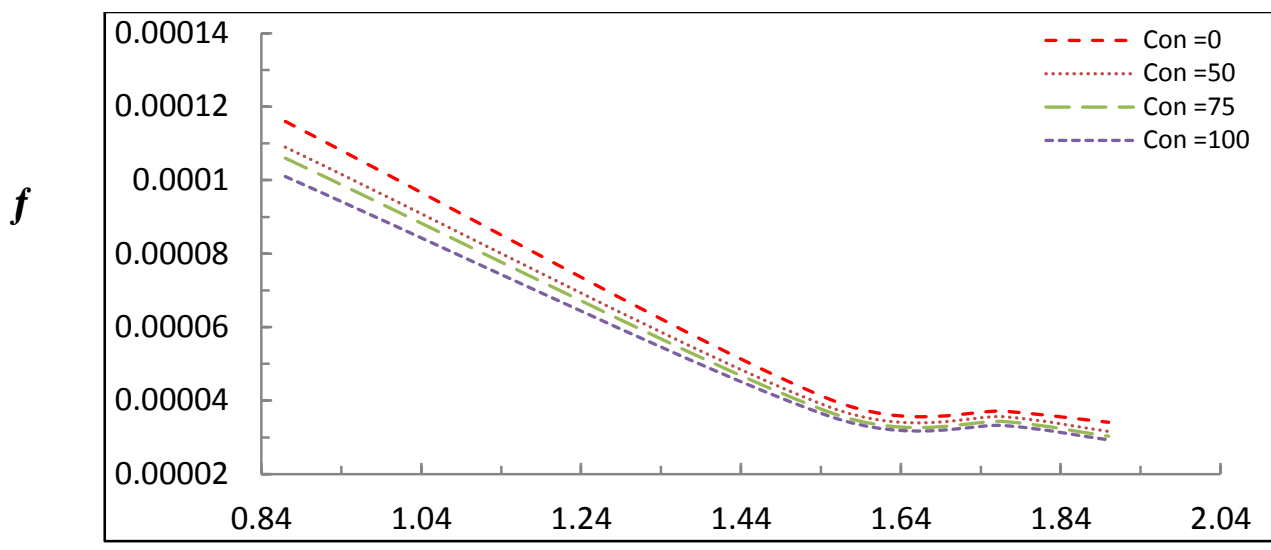

Velocity $(\mathrm{m} / \mathrm{s})$

Fig.6 friction factor with mean velocity for different polymer concentration $(\mathrm{ppm})$, at time $=90$ minutes

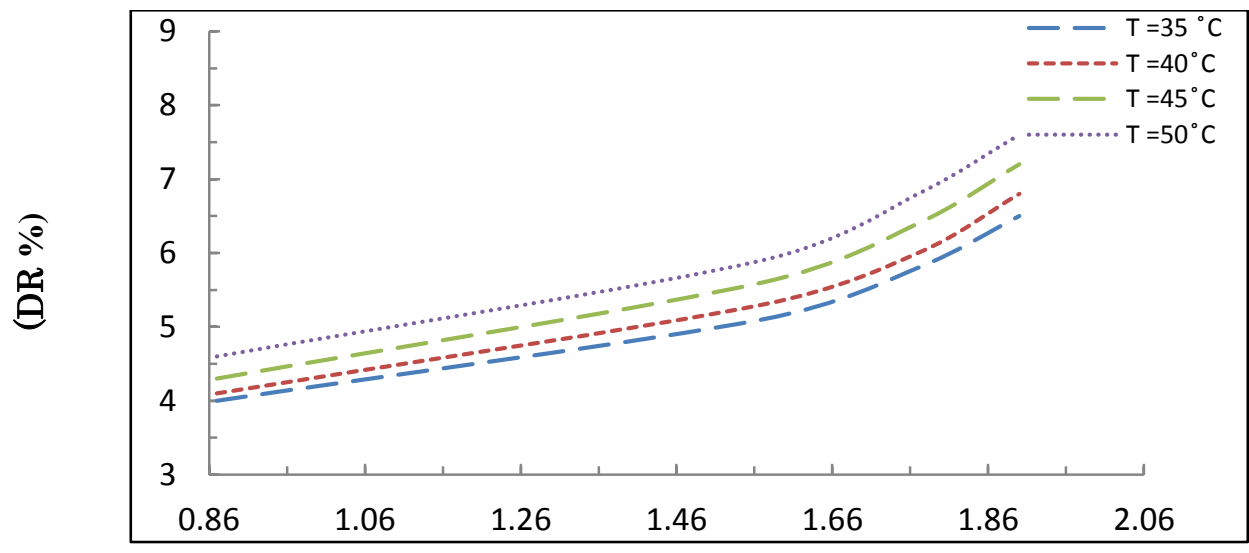

Velocity $(\mathrm{m} / \mathrm{s})$

Fig.7 effect of velocity on drag reduction percentage for different temperature at polymer $\mathrm{C}=50 \mathrm{ppm}$

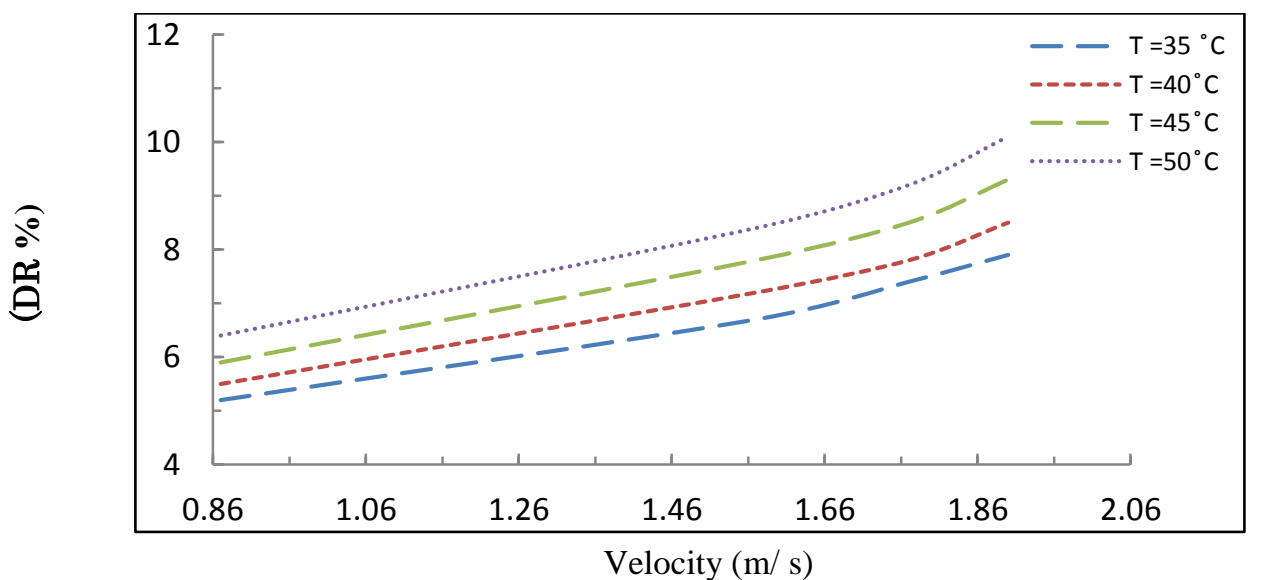

Fig. 8 effect of velocity on drag reduction percentage for different temperature at polymer $\mathrm{C}=75 \mathrm{ppm}$ 


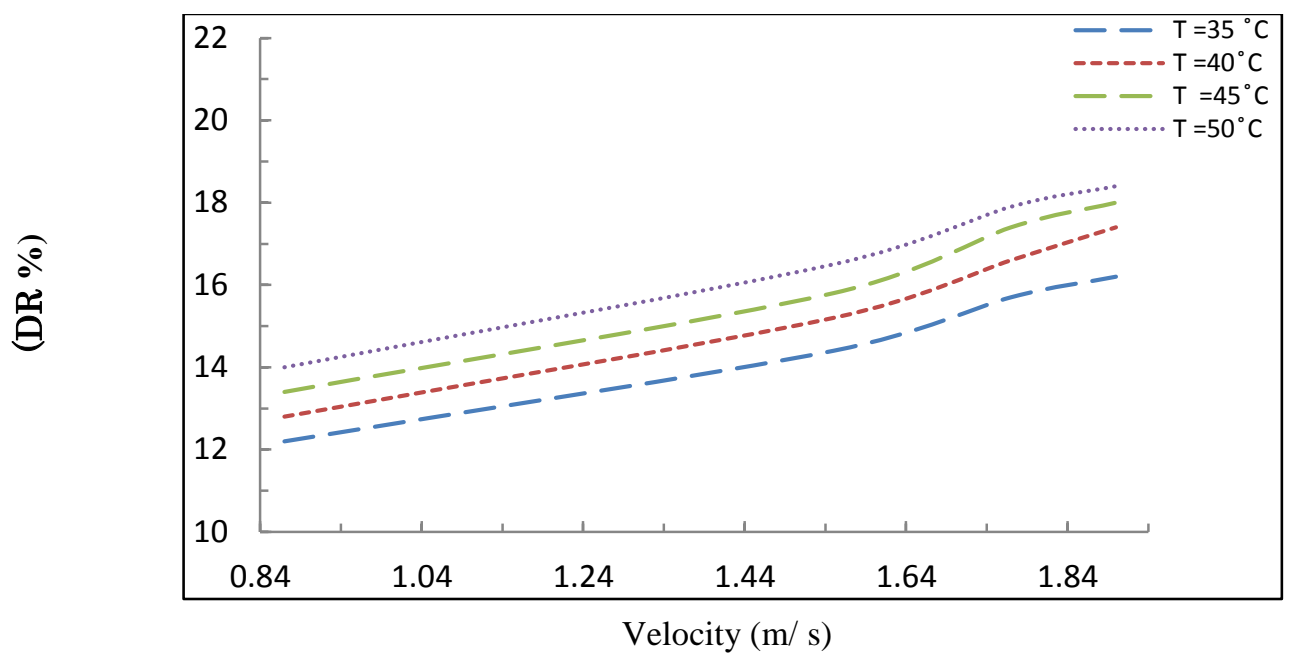

Fig.9 effect of velocity on drag reduction percentage for different temperature at polymer $\mathrm{C}=100 \mathrm{ppm}$

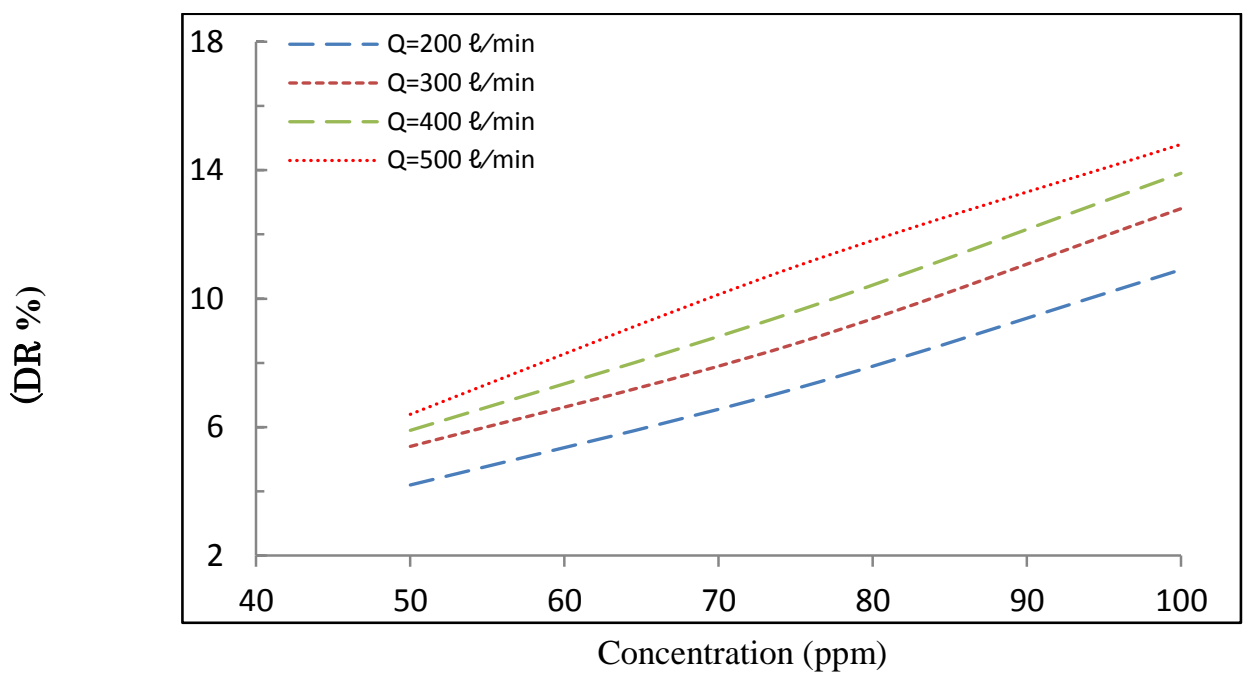

Fig.10 drag reduction with polymer concentration for different velocity, at time $=90$ minutes

\section{REFERENCES}

Neamah, A. H. , 2012 " Experimental and Theoretical Studies of Hot Plate Cooling by Jet Impingement", M.Sc. Thesis, Mechanical engineering, University of Babylon.

Cruz, D. O. A., Pinho. F. T. and Resende. P. R. , 2004 " Modeling the New Stress for Improved Drag Reduction Predictions of Viscoelastic Pipe Flow", J.of Non- Newtonian Fluid Mech., vol. 121, pp. 127-141. 
Min, T., Yoo, J., Choi, H., and Joseph, D. D., 2003 " Drag Reduction by Polymer Additives in a turbulent Channel Flow", J. of Fluid Mech., vol. 486, pp. 213- 238 .

Yuan, S.W., 1970 “foundations of Fluid Mechanics" , Book, Prentice-Hall, international Inc, S.I .Unit, first edition, London.

COMSOL 4.4 User Manual, www. COMSOL, Com.

Shahath, A. A., 2015, "Drag Reduction in Turbulent Pipe Flow Using Additives" , M.Sc. Thsis , Department of Mechanical Engineering, University of Babylon .

ChungYin , L. , 2011, "The Effect of Polymer Concentration on Turbulent Drag Reduction" , M.Sc. Thesis, The Chinese University of Hong Kong.

Paschkewitz, J. S. , Dubiet , Y. ,Dimitropoulos, C. D. ,Shaqfech, G. G., and Moin , P. , 2004 "Numerical Simulation of Turbulent Drag Reduction using Rigid Fibers", J. of Fluid Mech. Vol. 518, pp. 281-317.

Dubief, Y. , White , C. M. ,Terrapon , V. T. „Shaqfen , E. S. G., Moin , P. , and Level , S. K. , 2004 "on the Coherent Drag Reduction and Turbulent-Enhancing Behavior of Polymers in Wall Flows", J. of Fluid Mech. Vol. 514, pp.271-280.

Sher and Hetsroni , G. , 2008 " A mechanistic Model of Turbulent Drag Reduction By Additives " , J. of Chemical Engineering Science, Vol. 63 , pp. 1771-1778 .

Hadri , F. , and Gullou , S. , 2010 " Drag reduction by surfactant in Closed turbulent Flow" , International Journal of Engineering Science and Technology, Vol. 2 ,No. 12, pp. 6876-6879.

Mohammed, A.J, 2017, "Drag Reduction Pipe Flow with Polymer Injection" M.Sc. Thesis , Mechanical Engineering Department, University of Babylon, Unpublished. 